\title{
Fast Tracking of Evoked Potential Variations Using Correlated Scale Function Designed by Multiresolution Analysis
}

\author{
W. Q. Liu, F. H. Y. Chan, F. K. Lam, M. H. Du \\ Department of Electrical and Electronic Engineering, The University of Hong Kong \\ E-mail: fhychan@eee.hku.hk
}

\begin{abstract}
Fast tracking of evoked potential variations is of great importance in clinical operation. The paper describes a method whereby ensemble averaged signal is used as the prototype of the scale function and designs a correlated scale function based on multiresolution analysis. Hence, an effective low pass digital filter having powerful tracking capability is obtained. Results show that the filter designed filters out the noise more effectively than that of using general wavelet filtering, and the tracking of the peak of evoked potential is easily obtained.
\end{abstract}

Index Terms-Multiresolution, evoked potential, scale function, two scale relation.

\section{Introduction}

Evoked Potentials(EPs), which are brain electrical activities resulting from sensory stimulation play an important role in the diagnosis of the abnormalities of human brain. However the amplitude of the EP is a few tenths of a micro-volt, much smaller than that of the spontaneous EEG activity, and extensive averaging is required to obtain a reliable estimate of the response. Many techniques have been developed for extracting information from event-related potential. Ensemble averaging (EA) is the most widely used method for obtaining the EPs. However, this method ignores the fact that the EPs are also time-varying signals and the results of EA may introduce significant distortion and loss of information about the response variability from trial to trial. Adaptive filter shows promising in get almost real time response, but the response gotten by the adaptive filter is not the true response, for it obtains the "true" response by minimizing the difference between the real response and the reference input. However the reference input is usually set according to the past knowledge and is not the actual reflection of the changing EPs. Some researcher used wavelet transform to get rid of the noise by eliminating the unrelated detailed signals[6]. However, due to the low SNR of the signal, the results were not so satisfactory. Actually, many methods of bandpass filtering have been used to eliminate as much spontaneous EEG activity as possible without affecting the EP itself. But the filter design is complicated. In this paper, we use the EA signal of the evoked potentials as the prototype of the scale function(father wavelet), ensuring a high correlation between the scale function and the EP signal. By means of multiresolution analysis, the corresponding digital filter is easily obtained and fast tracking of the evoked potentials using the designed filter is accomplished.

\section{Methods}

Wavelet transform (WT) is widely used in the field of signal processing recently[3]. It has many advantages when compared with the traditional Short Time Fourier Transform (STFT). The window of STFT is fixed and loses some information when the signal to be analyzed changes very fast, while WT can adapt to the signal and can zoom into details of the signals. It also has simple algorithm and can process almost in real time the time-varying characteristic of signals. Besides, if the signal to be processed is real, then the decomposition and the reconstruction can be done in real function. WT divides every signal into two parts, with one in low frequency band and the other part in higher frequency one. From a view of filtering, one part is low-pass filtering and the other part is the band-pass filtering. In this paper, the wavelet representation proposed by Daubechies [5] is used here. Let $\phi(x)$ be a scale function and $\psi(x)$ be the corresponding wavelet. Defining the coefficients of filters $\mathrm{H}$ and $\mathrm{G}$ as follows,

$$
\left\{\begin{array}{l}
h(n)=2^{-1 / 2} \int d x \phi\left(\frac{1}{2} x\right) \phi(x-n) \\
g(n)=2^{-1 / 2} \int d x \psi\left(\frac{1}{2} x\right) \psi(x-n)
\end{array}\right.
$$

Suppose the data of the $i$-th trial of evoked potential is $\left\{x_{i}(0), x_{i}(1) \cdots x_{i}(N-1)\right\}$, and for clarity, let it be $\left\{c_{0}^{0}, c_{1}^{0} \cdots, c_{N-1}^{0}\right\}$, so we have the decomposition formula:

$$
\left\{\begin{array}{l}
c_{k}^{j}=\sum_{n} h(n-2 k) c_{n}^{j-1} \\
d_{k}^{j}=\sum_{n} g(n-2 k) c_{n}^{j-1}
\end{array}\right.
$$

where $j$ indicates the scale, $\left\{c_{k}^{j}\right\}$ is the coarse signal, and $\left\{d_{k}^{j}\right\}$ is detailed signal. The corresponding reconstruction formula is:

$$
\dot{c}_{n}^{j-1}=\sum_{k} h(n-2 k) c_{k}^{j}+\sum_{k} g(n-2 k) d_{k}^{j}
$$


Like Fourier decomposition, Wavelet decomposition views signal in different scales. In frequency domain, different scales consist of different frequencies although there is not a direct expression as that in the Fourier transform. Different scales account for different center frequency filters. If the wavelet transform is orthogonal (e.g. Daubechies wavelet), the filter is a quadrature filter. In many area of signal processing, this orthogonal decomposition will help to rearrange the signal such that processing of wanted information may be achieved. But the orthogonal wavelet transform is sensitive to the data acquired and in some applications, some redundancy is needed if the SNR of the sampled data is low. We can see that wavelet transform is to build a filter bank. Different basis function means different filter bank. In most of the application of signal processing, the main idea or the main work is to design a good filter so that the noise can be filtered out and leave only the desired information. In the field of weak signal extraction, it translates into SNR enhancement. In this paper, a correlated scale function is designed by means of multiresolution analysis, and is used as multiresolution variation tracking. Orthogonality is not crucial here in tracking.

Two scale relation[7] plays an important part in multiresolution analysis

$$
\phi(x)=\sum_{n=0}^{m} c_{n} \phi(2 x-n)
$$

where $c_{n}(n=0,1, \cdots, m)$ are coefficients.

We know that in the frequency domain, scale function is mainly with low frequency. So, we can use the ensemble averaging of the VEP signal as the prototype of scale function. By the theory of the multiresolution, the signal should satisfy the two scale relation. We use iteration algorithm to optimize the EA signal as the scale function. The details of calculating $c_{n}$ are as following:

Let the EA signal (Fig. 1) be the scale $\phi(x)$. Suppose we use totally $m+1$ coefficients $c_{0}, c_{1}, c_{2}, \ldots, c_{m}$, we select the value so that the following is minimum (least square error):

$$
\lambda=\left[\phi(x)-\sum_{n=0}^{m} c_{n} \phi(2 x-n)\right]^{2}
$$

Since all the calculations are executed by a computer, we use discrete expressions.

Let $\phi(x)=\left(\begin{array}{c}\phi_{1} \\ \phi_{2} \\ \vdots \\ \phi_{n}\end{array}\right), \phi(2 x-k)=\left(\begin{array}{c}x_{1 k} \\ x_{2 k} \\ \vdots \\ x_{n k}\end{array}\right)$ where $k=1,2, \cdots, n$.

Then we have

$$
\left(\begin{array}{c}
\phi_{1} \\
\phi_{2} \\
\vdots \\
\phi_{n}
\end{array}\right)=c_{0}\left(\begin{array}{c}
x_{10} \\
x_{20} \\
\vdots \\
x_{n 0}
\end{array}\right)+c_{1}\left(\begin{array}{c}
x_{11} \\
x_{21} \\
\vdots \\
x_{n 1}
\end{array}\right)+\cdots+c_{m}\left(\begin{array}{c}
x_{1 m} \\
x_{2 m} \\
\vdots \\
x_{n m}
\end{array}\right)
$$

To select the best $c_{0}, c_{1}, \ldots, c_{m}$, we should minimize the following $\lambda$ :

$$
\lambda=\sum_{i=1}^{n}\left(\phi_{i}-\sum_{k=0}^{m} c_{k} x_{i k}\right)^{2}
$$

From $\partial \lambda / \partial c_{l}=0$, we get

$$
\sum_{i=1}^{n}\left(\phi_{i}-\sum_{k=0}^{m} c_{k} x_{i k}\right) x_{i l}=0 \quad l=0,1, \cdots, m .
$$

So

$$
\sum_{k=1}^{m}\left(\sum_{i=1}^{n} x_{i k} x_{i l}\right) c_{k}=\sum_{i=1}^{n} \phi_{i} x_{i l} \quad l=0,1, \cdots, m .
$$

With (6) we could solve the $c_{0}, c_{1}, \ldots, c_{m}$.

We know that $\sum_{k=0}^{m} c_{k}=2$, for multiresolution scale function $\varphi(x)$ [7]. Because the raw EA signal $\phi(x)$ is not a function constructed by multiresolution, the sum of $c_{0}, c_{1}, \ldots, c_{m}$ may deviate from 2 . We use iteration algorithm to achieve a function with the sum of $c_{0}, c_{1}, \ldots, c_{m}$ approaching 2 .

That is , after solving $c_{0}, c_{1}, \ldots, c_{m}$, we use

$$
c_{0}\left(\begin{array}{c}
x_{10} \\
x_{20} \\
\vdots \\
x_{n 0}
\end{array}\right)+c_{1}\left(\begin{array}{c}
x_{11} \\
x_{21} \\
\vdots \\
x_{n 1}
\end{array}\right)+\cdots+c_{m}\left(\begin{array}{c}
x_{1 m} \\
x_{2 m} \\
\vdots \\
x_{n m}
\end{array}\right) \text { as a new }\left(\begin{array}{c}
\phi_{1} \\
\phi_{2} \\
\vdots \\
\phi_{n}
\end{array}\right) \text {. }
$$

Redo the above (6), until we get $c_{0}, c_{1}, \ldots, c_{m}$, with their sum approximately equal to 2 . In this paper, the criteria of selecting $c_{0}, c_{1}, \ldots, c_{m}$, is $\left|\sum_{k=0}^{m} c_{k}-2\right| \leq 10^{-2}$.

\section{Implementation}

We use the designed scale function to analyze the VEP signal. We know that the most important thing in VEP signal is its latency and the corresponding amplitude[1],[2]. In this paper, the main thing is to track the peak, so the 
orthogonality of transform is not so important as in the data compression. In clinical operation, the main task is to monitor the latency shifting and its amplitude variation in real time, real time analysis is desired. On account of the need for fast tracking, we use 17 coefficients to construct a scale function.

The EA VEP signal (Fig. 1) was used as the prototype of scale function. Using the method mentioned above, we get

$\mathrm{Cn}=\left[\begin{array}{llllll}0.0753 & 0.0324 & 0.0638 & 0.0430 & 0.2593 & 0.2120\end{array}\right.$

$\begin{array}{lllllll}0.3715 & 0.3048 & 0.1797 & 0.3531 & 0.0438 & -0.1136\end{array}$

$\left.\begin{array}{lllll}0.1149 & -0.0882 & -0.0044 & -0.0396 & 0.1822\end{array}\right]$

where the sum of the coefficients is 1.99 .

The construction of scale function is shown in Fig. 2. We know that

$$
\phi(x)=\sqrt{2} \sum_{n=0}^{m} h_{n} \phi(2 x-n) .
$$

A digital filter $\mathrm{H}$ is obtained by setting

$$
h_{n}=c_{n} / \sqrt{2}(n=0,1, \cdots, m) .
$$

The frequency response of the filter $\mathrm{H}$ is

$$
H\left(e^{j \omega}\right)=\sum_{k=0}^{m} h_{k} e^{-j \omega k} .
$$

$\left|H\left(e^{j \omega}\right)\right|$ is shown in Fig. 3. Compared with that of Daubechies 20 coefficients filter( Fig. 4), the constructed filter is more complicated, due to the requirement of high correlation with the EA VEP signals. Nevertheless, the construction of the digital filter is still simple.

The multiresolution analysis using the designed filter is shown in Fig. 5. And Fig. 6 shows the corresponding results using Daubechies wavelet with 24 coefficients. The tracking method proposed by Liu [4] was used. It is obvious that the multiresolution tracking in [4] is easily implemented by the designed filter in this paper.

\section{Conclusion}

We can see that the designed scale function filters can track the waveform of the evoked potential more effective than that of Daubechies wavelets. This is because the designed scale function has high correlation with the evoked potential. We know that the designed scale function is derived from the EA signal( prototype signal). So different patients require different filters. Furthermore, we can construct different filters during different periods of time for the same patient. Due to its simple construction and effectiveness in extracting the waveform of the evoked potential, the scale function is specially useful for fast tracking in clinical operation, where a prompt response should be made while there is any sign of abnormality during the operation. The method mentioned above is also suitable for SEP peak tracking.

\section{Acknowledgement}

The authors would like to thank the Research Council, Hong Kong Special Administrative Region Government for financial assistance.

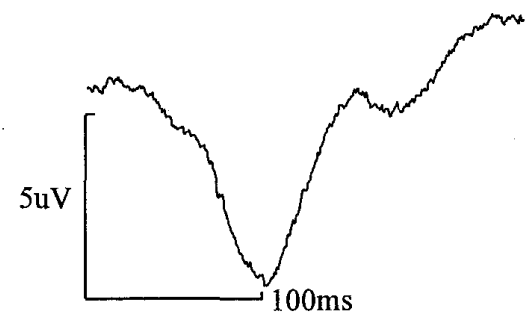

Fig. 1 The EA VEP signal for constructing the scale function.

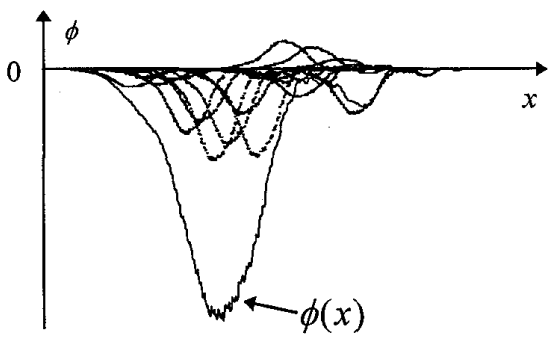

Fig. 2. The construction of correlated scale function.

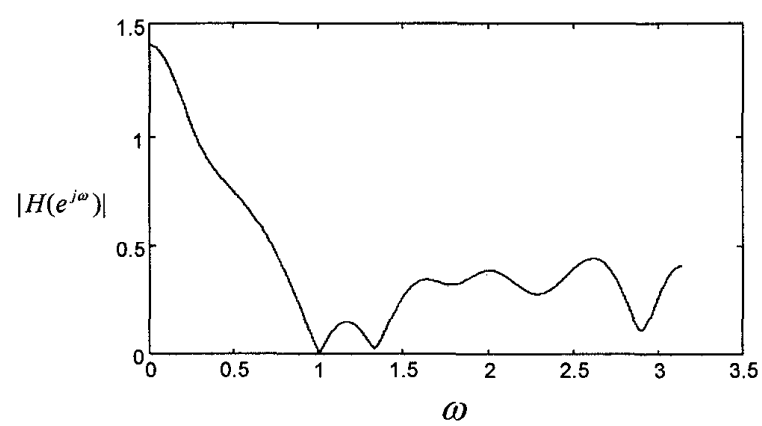

Fig 3. The frequency response $\left|H\left(e^{j \omega}\right)\right|$ of the designed filter. 


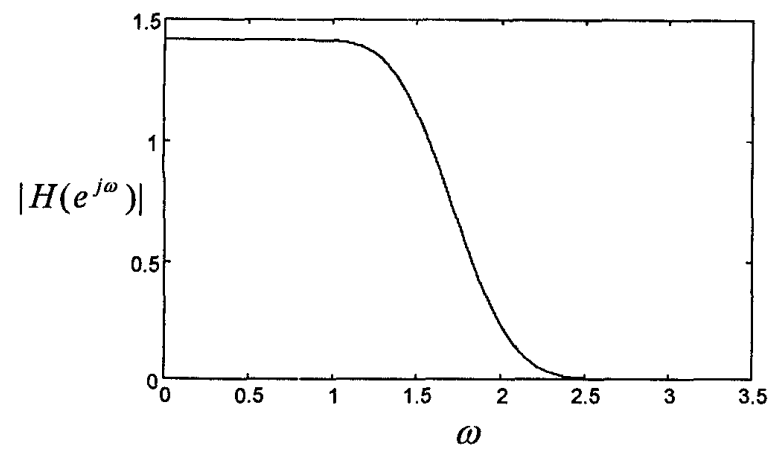

Fig 4. The frequency response $\left|H\left(e^{j \omega}\right)\right|$ of Daubechies' scale function.

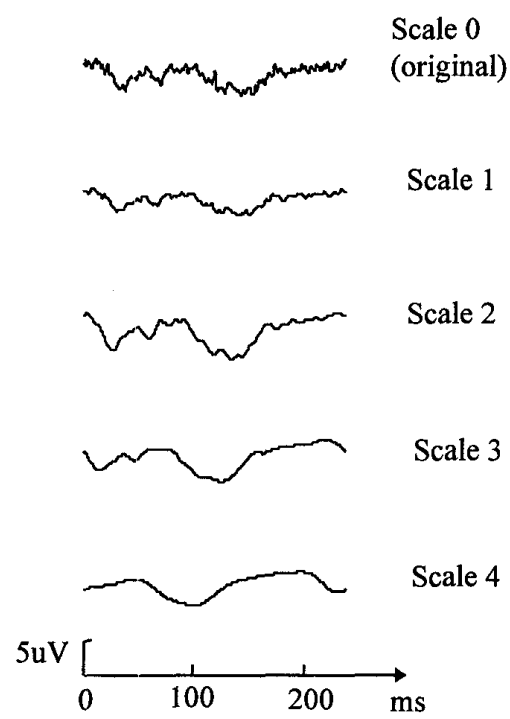

Fig. 5. Multiresolution tracking using the designed scale function, tracking from large scale (bottom).

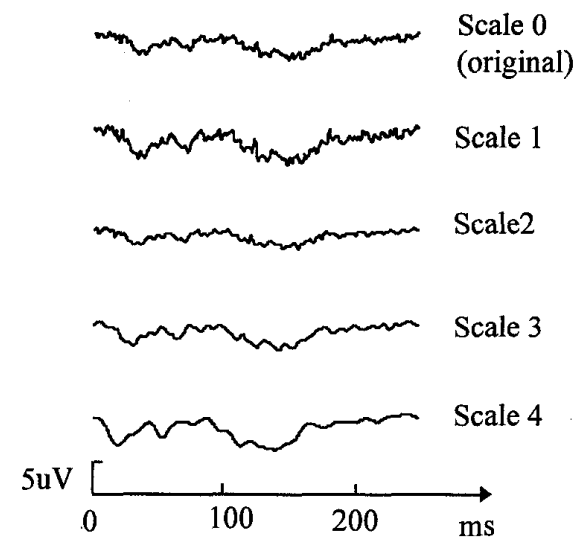

Fig. 6. Multiresolution tracking using Daubechies's 20 coefficients wavelet, tracking from large scale (bottom).

\section{References}

[1] D. Regan, Human brain electrophysiology: evoked potentials and evoked magnetic fields in science and medicine, Elsevier, New York, 1989.

[2] Rafael E. Delgado, özcan özdamar, "Automated auditory brainstem response interpretation", IEEE Engineering in Medicine and Biology, April/May 1994.

[3] S. G. Mallat, "A theory for multiresolution signal decomposition: the wavelet representation", IEEE Trans. Pattern Analysis and Machine Intelligence, Vol. 11 No.7, July, 1989.

[4] W. Q. Liu , F.H.Y. Chan, F.K. Lam and P.W.F. Poon, "Wavelet based methods for tracking the latency of evoked potentials", Proc. of 17th EMBS conference, pp. 1061-1062, 1995.

[5] 1. Daubechies, "Orthonormal bases of compactly supported wavelets," Commun. Pure Appl. Math.. vol. XLI, pp.909-996,1988.

[6] E. A. Bartnik, K.J. Blinowska and P. J. Durka, "Sigle evoked potential reconstruction by means of wavelet transform", Biol. Cybern. 67, pp 175-181, 1992.

[7] Charles K. Chui, "A introduction to wavelets", Boston: Academic Press, 1992. 